\title{
Synthesis of heteroaryl triazenes via Rh(III)-catalyzed annulation reactions with alkynyl triazenes.
}

\author{
Tim Wezeman, ${ }^{\mathrm{a}}$ Rosario Scopelliti, ${ }^{\mathrm{a}}$ Farzaneh Fadaei Tirani, ${ }^{\mathrm{a}}$ and Kay Severin ${ }^{\mathrm{a}, *}$ \\ a Institut des Sciences et Ingénierie Chimiques, Ecole Polytechnique Fédérale de Lausanne (EPFL), 1015 Lausanne, \\ Switzerland. \\ Email: kay.severin@epfl.ch.
}

Received: ((will be filled in by the editorial staff))

Supporting information for this article is available on the WWW under http://dx.doi.org/10.1002/adsc.201

\begin{abstract}
A synthetic route towards polycyclic heteroaryl triazenes has been developed. Alkynyl triazenes were coupled to phenyl-substituted indoles, imidazoles and sydnones via rhodium-catalyzed double C-H or C-H/N-H activation reactions. The oxidative annulations gave triazenes connected to imidazo[1,2-a]quinoline, indolo[2,1-a]isoquinoline and sydnone-quinoline scaffolds in moderate to excellent yields. The triazene-bearing fused sydnone-quinolines were used for subsequent orthogonal transformations. The sydnone moiety allowed for post-functionalization via 1,3-dipolar cycloadditions in excellent yields, and the triazene group can be substituted by a range of functional groups.
\end{abstract}

Keywords: Rhodium catalysis; C-H/N-H activation; Triazene; Sydnone.

\section{Introduction}

Aryl triazenes have been used as linkers in solidphase synthesis, ${ }^{[1]}$ as directing groups in $\mathrm{C}-\mathrm{H}$ activation reactions, ${ }^{[2]}$ as protecting group for amines in complex syntheses, ${ }^{[3]}$ and as precursor to diazonium salts and a wide variety of functional groups. ${ }^{[4]}$ Besides utilization as reagents in synthetic chemistry, triazenes can also exhibit interesting biological activities, with two triazenes (dacarbazine and temozolomide) being used as chemotherapy agents, and several others being evaluated for their anti-tumor activity. ${ }^{[5]}$

1-Aryl triazenes are commonly prepared from functionalized aromatic compounds $\operatorname{ArX}$ (Scheme 1a). The triazene group is then installed by using an appropriate coupling chemistry. The most frequently employed procedure involves the coupling of aryldiazonium salts with secondary amines. $\left.{ }^{[1,} 4\right]$ Alternatively, it is possible to build the triazene function from aromatic nitroso compounds by coupling with hydrazines, ${ }^{[6]}$ or from aryl Grignard reagents by coupling with azides ${ }^{[7]}$ or aminodiazotates. $^{[8]}$

We have recently described a protocol for the synthesis of 1-alkynyltriazenes. ${ }^{[8]}$ Due to the presence of the triazene function, the alkyne is activated towards electrophilic attack at the $\beta$ position. As a consequence, 1-alkynyltriazenes display a reactivity profile similar to that of ynamides. ${ }^{[9]}$

Having access to 1-alkynyl triazenes, we wanted to explore if it is possible to prepare 1-aryl triazenes by cyclization or annulation reactions (Scheme 1b). A first success in this direction was the synthesis of a triazene with a benzofuryl group in 1-position by iodocyclization of a 2-methoxyphenyl-substituted alkynyl triazene. However, the scope of this reaction is limited.

$$
\begin{aligned}
& \text { a) }
\end{aligned}
$$

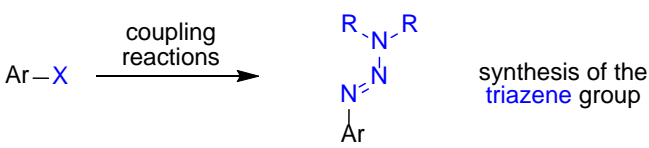

$$
\begin{aligned}
& \text { b) } \quad R_{-N}^{1}, R^{1}
\end{aligned}
$$

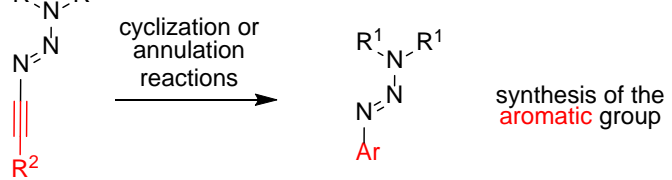

Scheme 1. Strategies for the synthesis of 1-aryl triazenes.

Transition metal-catalyzed annulation/cyclization reactions represent a potentially more versatile way to convert 1-alkynyl triazenes into 1-aryl triazenes, and our group has recently started to explore this option. Below, we show that $\mathrm{Rh}^{\mathrm{III}}$-catalyzed $\mathrm{C}-\mathrm{H}$-and $\mathrm{N}-\mathrm{H}$ activation reactions ${ }^{[10]}$ can be used to synthesize a range of polycyclic heteroaryl triazenes. Furthermore, 
we show that the triazene function in the products can be used for subsequent synthetic transformations.

\section{Results and Discussion}

We started our investigations by testing the double $\mathrm{C}-\mathrm{H}$ activation of $\mathrm{N}$-phenylimidazole (2) in the presence of alkynyl triazene $\mathbf{1}$ under typical literature conditions (toluene, $110{ }^{\circ} \mathrm{C}, \mathrm{Cu}(\mathrm{OAc})_{2}$ as oxidant) ${ }^{[11]}$ with $\left[\mathrm{Cp}^{*} \mathrm{RhCl}_{2}\right]_{2}$ (5 mol\% of the complex, $10 \mathrm{~mol} \%$ $\mathrm{Rh})$ as catalyst precursor (Table 1 , entry 1$).{ }^{[12]}$ We observed full conversion of $\mathbf{1}$ within a few hours and the formation of a new triazene-containing compound. However, the analytical data showed that we had isolated vinyl triazene $\mathbf{4}$ instead of the desired azafused quinoline 3 .

Compound $\mathbf{4}$ is known to form by addition of acetic acid to triazene $\mathbf{1}$ at elevated temperatures. ${ }^{\text {[9a] }}$ Therefore, we decided to perform a small screening of reactions conditions at lower temperatures. $\mathrm{CD}_{3} \mathrm{CN}$ was used as solvent, allowing to follow the reactions by ${ }^{1} \mathrm{H}$ NMR spectroscopy.

Table 1. Rh-catalyzed reaction of 1-phenylimidazole with alkynyl triazene $\mathbf{1}$.
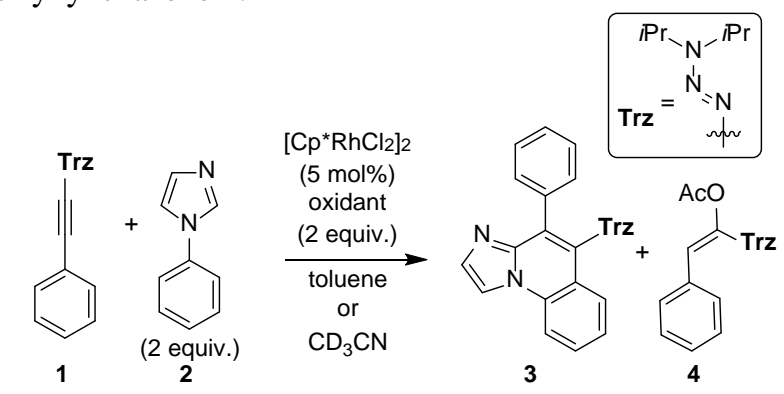

\begin{tabular}{|c|c|c|c|c|c|c|}
\hline entry & $\begin{array}{c}\text { temp } \\
\left({ }^{\circ} \mathrm{C}\right)\end{array}$ & tim & & base & con & $\begin{array}{l}\text { ratio } \\
(3: 4)\end{array}$ \\
\hline 1 & $110^{b}$ & $4 \mathrm{~h}$ & $\mathrm{Cu}(\mathrm{OAc})_{2}$ & - & $>95 \%$ & $\sim 1: 20$ \\
\hline 2 & 80 & $12 \mathrm{~h}$ & $\mathrm{Cu}(\mathrm{OAc})_{2}$ & - & $>95 \%$ & $5: 6$ \\
\hline 3 & 80 & $12 \mathrm{~h}$ & AgOAc & - & $>95 \%$ & $10: 9$ \\
\hline 4 & 80 & $24 \mathrm{~h}$ & AgOAc & $\mathrm{Na}_{2} \mathrm{CO}_{3}$ & $42 \%$ & $5: 1$ \\
\hline 5 & 60 & $3 d$ & AgOAc & - & $>95 \%$ & $3: 2^{c}$ \\
\hline 6 & 80 & $12 \mathrm{~h}$ & $\mathrm{Cu}(\mathrm{OAc})_{2}$ & $\mathrm{Na}_{2} \mathrm{CO}_{3}$ & $>95 \%$ & $4: 1^{d}$ \\
\hline
\end{tabular}

a) Determined by ${ }^{1} \mathrm{H}-\mathrm{NMR}$ spectroscopy. ${ }^{\text {b) }}$ Toluene was used as solvent. ${ }^{c)}$ Isolated yield of 3: 38\%. ${ }^{d)}$ Isolated yield of 3: $62 \%$. Trz = 3,3-diisopropyltriaz-1-ene.

Lowering the reaction temperature from $110{ }^{\circ} \mathrm{C}$ to $80{ }^{\circ} \mathrm{C}$ partly mitigated side product formation (Table 1 , entries 2 and 3), and allowed detection of the desired product in significant amounts. Addition of the base sodium carbonate reduced the rate at which the side product was formed, but also hindered completion of the reaction (Table 1, entry 4). Lowering the reaction temperature to $60{ }^{\circ} \mathrm{C}$ and increasing the reaction time to 3 days resulted in the formation of $\mathbf{3}$ as the dominant product. From a reaction performed on a preparative scale, we were able to isolate the desired product in $38 \%$ yield in the form of a single regioisomer (Table 1 , entry 5). Using copper acetate in the presence of sodium carbonate resulted in full conversion with a reasonable product to side product ratio (Table 1 , entry 6 ). Using these optimized conditions on a preparative scale we were able to isolate the product in $62 \%$ yield. Further screening of reaction conditions and reagents (including acetate-free oxidants and other bases) did not provide improved yields (see Table S-1 in Supporting Information, SI).

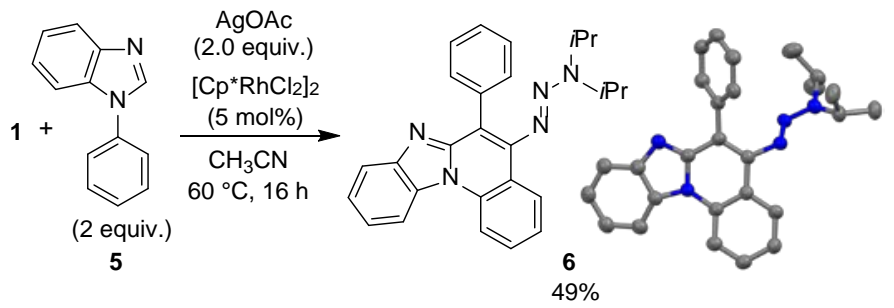

Scheme 2. Rh-catalyzed reaction of 1-phenylbenzimidazole with alkynyl triazene $\mathbf{1}$.

When $N$-phenylbenzimidazole ${ }^{[13]}$ (5) was used instead of $N$-phenylimidazole (2), we found that the reaction did not benefit from the addition of base and copper acetate, and the best yield was obtained using silver acetate at $60{ }^{\circ} \mathrm{C}$ without base. Using silver acetate we still observed the formation of side product 4, but were able to isolate the coupling product 6 in $49 \%$ yield (Scheme 2 ). The use of copper acetate in this reaction sped up the reaction, but mostly led to the increased formation of $\mathbf{4}$. In this case, the addition of base helped only slightly. The regioselectivity of the reaction was established by a crystallographic analysis of 6 .

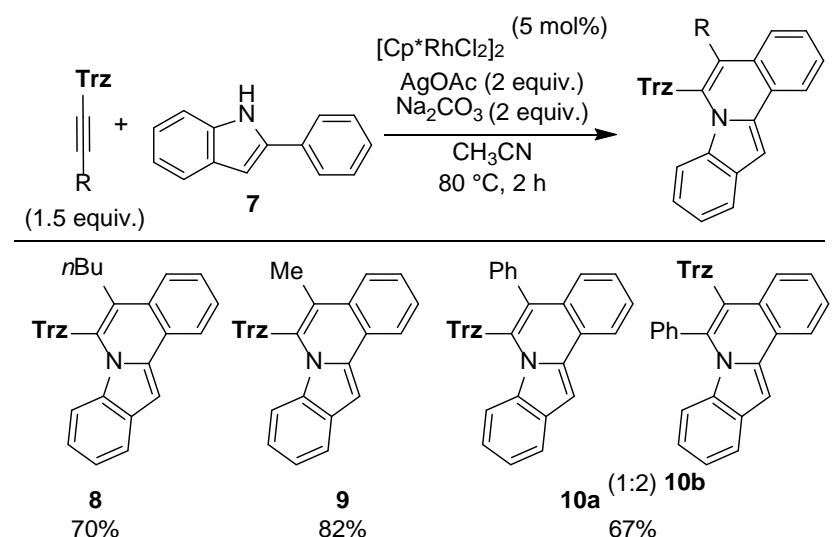

Scheme 3. Rh-catalyzed reactions of 2-phenyl-1H-indole with alkynyl triazenes. Trz = 3,3-diisopropyltriaz-1-ene.

Realizing that mild reactions conditions are crucial for avoiding side reactions of the alkynyl triazene, we screened for other substrates which could be annulated rapidly at lower temperatures. Indoles can undergo dual C-H and N-H activations. ${ }^{[14]}$ Although the typical conditions for $\mathrm{Rh}^{\mathrm{III}}$-catalyzed reactions between phenylindoles and alkynes often include elevated 
temperatures and moderately long reaction times, ${ }^{[14]}$ we observed that 2-phenyl-1H-indole reacted with ease with alkynyl triazenes at moderate temperatures. After a short optimization study (see SI), we found that using silver acetate in acetonitrile and column chromatography with basic aluminum oxide gave consistently the best yields. These conditions allowed synthesizing the annulation products 8-10 in good

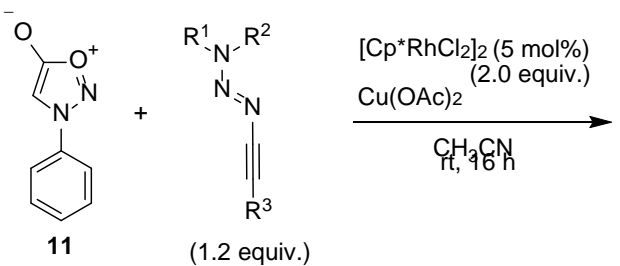

yields (Scheme 3). It is interesting to note that using an alkynyl triazene with an alkyl group attached to the triple bond led to the formation of a single regioisomer (confirmed by 2D NMR spectroscopy and X-ray crystallography), whereas the use of an alkynyl triazene with a phenyl group led to the formation of a 2:1 mixture of regioisomers.

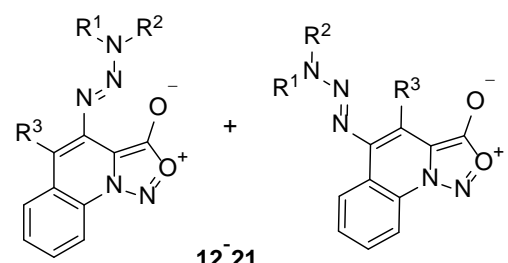

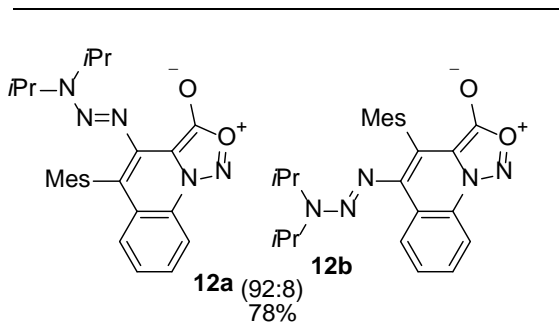

$$
\mathrm{Cy}-\mathrm{N}^{\mathrm{Cy}} \mathrm{O}
$$

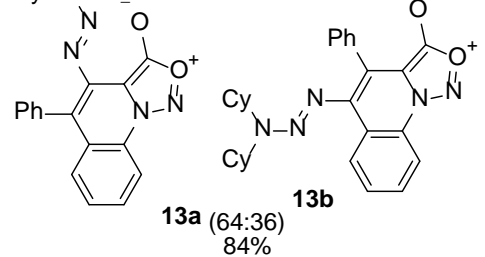<smiles>CC(C)N(/N=N/c1c(-c2ccccc2)c2ccccc2n2n[o+]c([O-])c12)C(C)C</smiles>
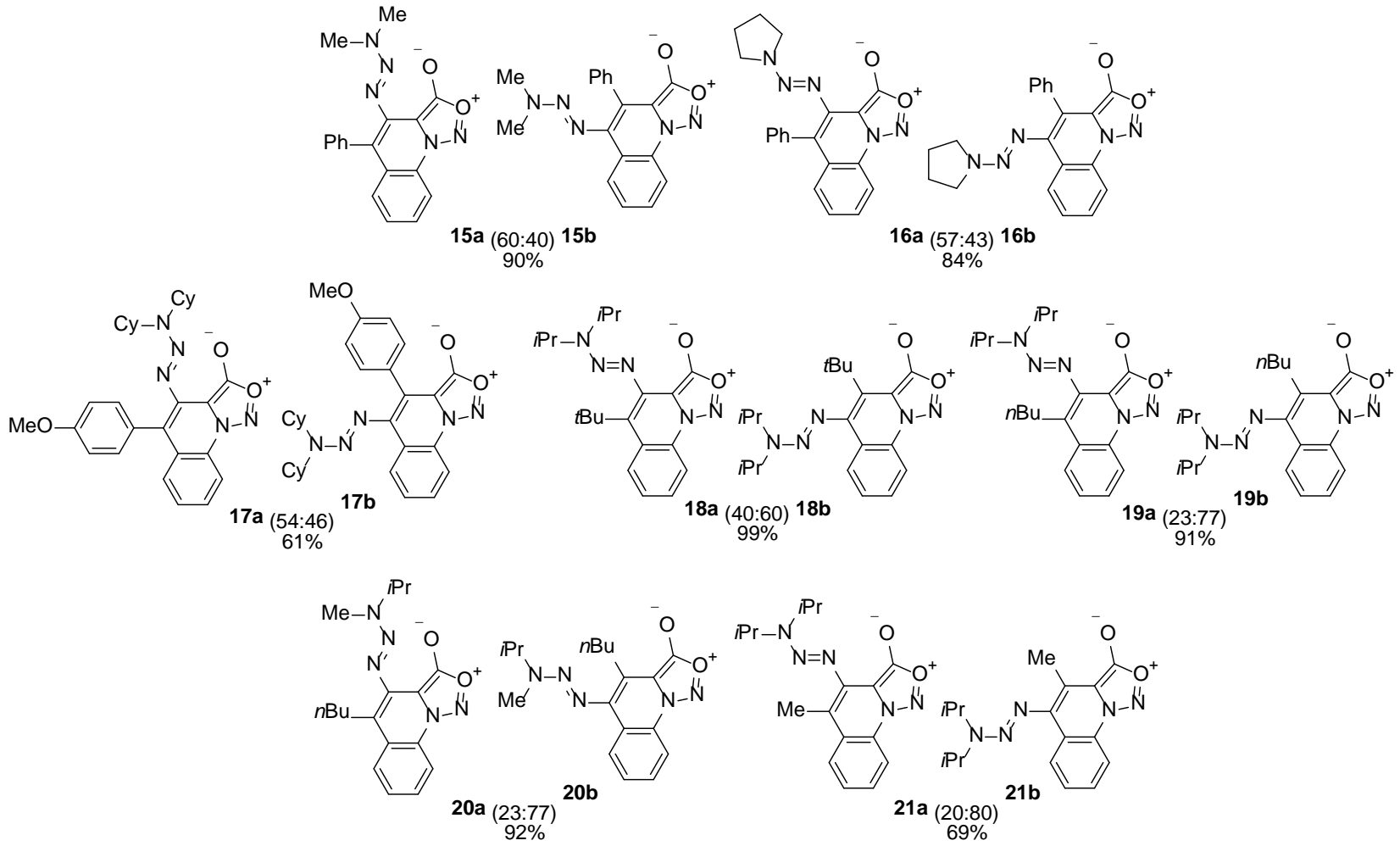

Scheme 4. Rh-catalyzed reaction of phenylsydnone with alkynyl triazenes. Isolated yields of both regioisomers are given, and the values in brackets indicate the ratio of the isomers. All isomers were separable by conventional column chromatography. Mes = mesityl.

Inspired by the recent work of Li and Wang, ${ }^{[15]}$ we investigated the use of $N$-phenylsydnone as substrate for $\mathrm{Rh}^{\mathrm{III}}$-catalyzed $\mathrm{C}$-H activation reactions with alkynyl triazenes. We found that these reactions can be performed at room temperature using $\mathrm{Cu}(\mathrm{OAc})_{2}$ as the oxidant. As a consequence of the mild reactions conditions, side products arising from degradation of the alkynyl triazenes were no longer observed. The resulting coupling products 12-21 were observed in good to excellent yields (Scheme 4). All reactions gave a mixture of regioisomers that were easily separable using conventional column chromatography. The ratio between the regioisomers was found to depend on the substrate, with the dominant factor being the group $\mathrm{R}^{3}$ attached to the triple bond of the alkynyl triazene (Scheme 4). By studying the kinetic isotope effect for 
the reaction between $N$-phenylsydnone and diphenylacetylene, $\mathrm{Li}$ and Wang concluded that $\mathrm{C}-\mathrm{H}$ bond cleavage of the sydnone is likely involved in the turnover-limiting step. ${ }^{[15]}$ We assume that a similar situation is found for reactions with alkynyl triazenes.

The key advantage of using alkynyl triazenes as substrates in cyclization/annulation reactions is the fact that the triazene function in the products can be used for further functionalizations. In the case of 12-21, there is the unique opportunity to use the triazene function $^{[4]}$ and the sydnone group ${ }^{[16]}$ for subsequent transformations. Since the chemical reactivity of these two groups is largely orthogonal to each other (Figure 1), we expected that we could perform diversification reactions in a site-selective fashion.

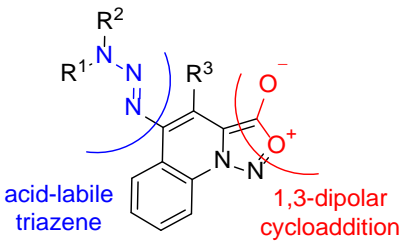

Figure 1. Orthogonal functionalization.

First, we set out to test the reactivity of the sydnone group using 14a and 14b as reactants (Scheme 5). Thermal and strain-promoted 1,3-dipolar cycloaddition reactions were found to proceed smoothly to give the pyrazolo[1,5-a]quinolone derivatives 22, 23, and 25. ${ }^{[16 a, 17]}$ Coupling of 14a to $\mathrm{N}$-ethylmaleimide ${ }^{[15,18]}$ led to the formation of bisadduct 24 in $82 \%$ yield. It is worth noting that the triazene function was not affected by all these coupling reactions.

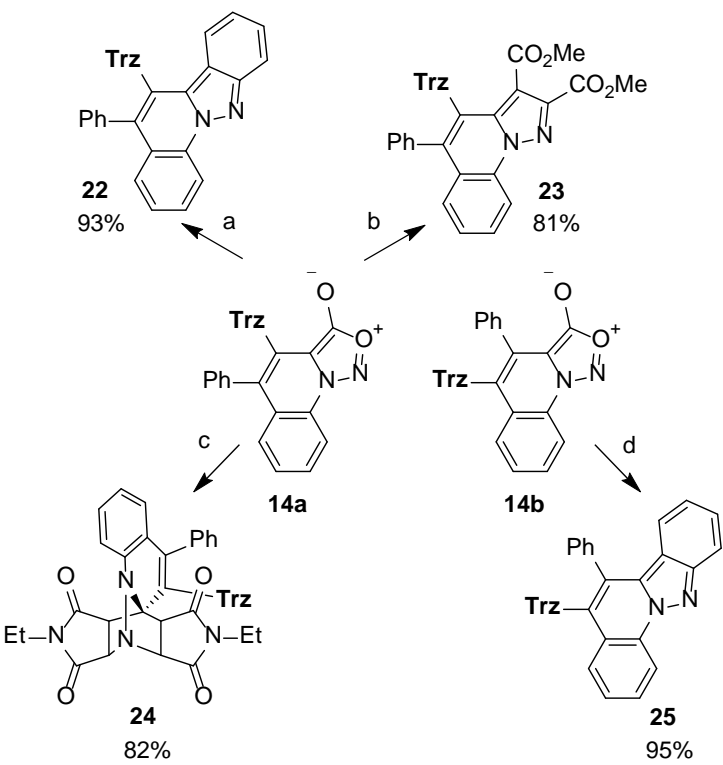

Scheme 5. Sydnone reactivity. Reaction conditions: a) 2(TMS)phenyl triflate, TBAF, THF, rt, 16 h; b) Dimethyl acetylenedicarboxylate, toluene, reflux, $16 \mathrm{~h}$; c) $\mathrm{N}$ Ethylmaleimide, toluene, reflux, 16 h; d) 2-(TMS)phenyl triflate, TBAF, THF, rt, 16 h. Trz = 3,3-diisopropyltriaz-1ene.
Next, we explored if we could selectively substitute the triazene function. As representative test reaction, we chose the acid-induced conversion of the triazene group into an arylazo group. As test substrates, we employed again 14a and 14b. The addition of $\mathrm{HBF}_{4}$ to a dichloromethane solution containing the respective triazene and an excess of an electron-rich arene lead to the clean formation of the azo compounds 26-29 (Scheme 6). In the case of $N, N$-dimethylaniline, the selective formation of the para isomer was observed.
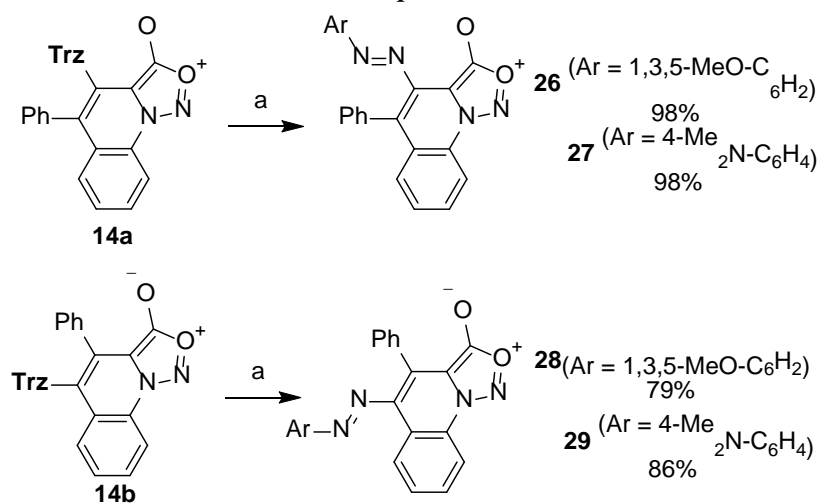

Scheme 6. Azo-coupling reactions. Reaction conditions: $\mathrm{HBF}_{4} \cdot \mathrm{Et}_{2} \mathrm{O}$, arene (2-5 equiv.), $\mathrm{CH}_{2} \mathrm{Cl}_{2}, 0{ }^{\circ} \mathrm{C}$ to rt, $16 \mathrm{~h}$.

Trz $=3$,3-diisopropyltriaz-1-ene.

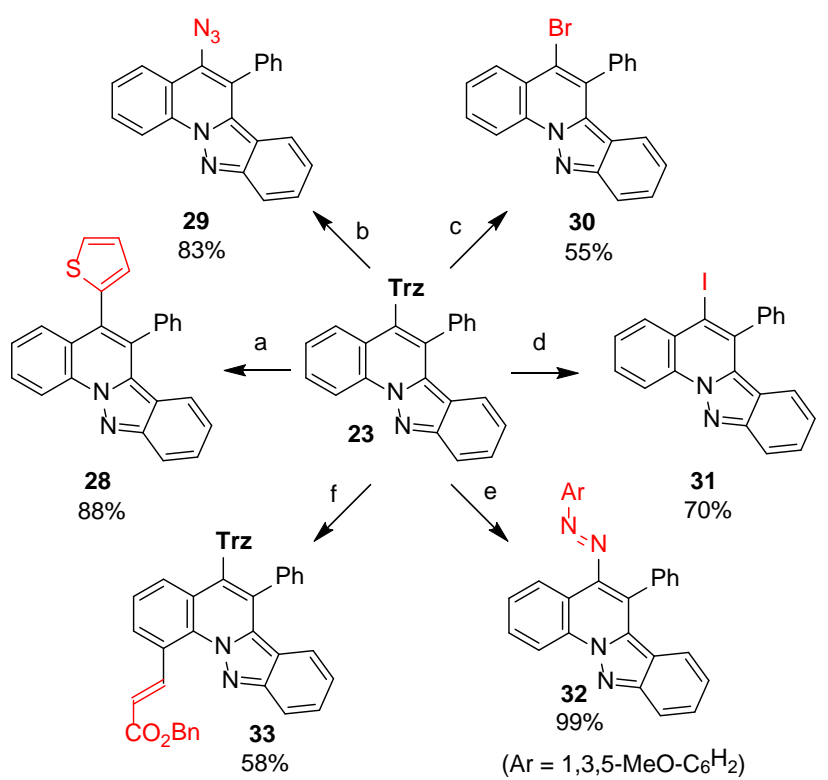

Scheme 7. Reactions of triazene 23. Reaction conditions: a) TFA, thiophene, $50{ }^{\circ} \mathrm{C}, 2 \mathrm{~h}, 88 \%$, r.r. $=4: 1$, main isomer shown; b) $\mathrm{HBF}_{4} \cdot \mathrm{Et}_{2} \mathrm{O}$, TMS-N $3, \mathrm{CH}_{2} \mathrm{Cl}_{2}, 0{ }^{\circ} \mathrm{C}$ to rt., $1 \mathrm{~h}$, $83 \%$; c) $\mathrm{HBr}$ (aq.), $\mathrm{CuBr}, \mathrm{Et}_{2} \mathrm{O}, 50{ }^{\circ} \mathrm{C}, 2 \mathrm{~h}, 55 \%$; d) $\mathrm{I}_{2}$, dichloroethane, $50{ }^{\circ} \mathrm{C}, 2 \mathrm{~h}, 70 \%$; e) $\mathrm{HBF}_{4} \cdot \mathrm{Et}_{2} \mathrm{O}$, 1,3,5trimethoxybenzene, $\mathrm{CH}_{2} \mathrm{Cl}_{2}, 0{ }^{\circ} \mathrm{C}$ to rt., $16 \mathrm{~h}, 99 \%$; ) AgOAc (0.3 equiv.), $\mathrm{Cu}(\mathrm{OAc})_{2}$ (2.0 equiv.), benzyl acrylate (2.5 equiv.), [Cp* $\left.\mathrm{RhCl}_{2}\right]_{2}$ (5 mol\%), $\mathrm{MeOH}, \mathrm{rt}, 16$ h, 58\%. Trz = 3,3-diisopropyltriaz-1-ene.

The fact that it is possible to use both the triazene and the sydnone group for synthetic transformations makes the annulation products 12-21 interesting starting 
materials for the diversity-oriented synthesis of polycyclic heteroaromatic compounds.

To demonstrate the versatility of the triazene group in this context, we have performed a series of substitution reactions with the indazolo[2,3a]quinolone $\mathbf{2 5}$. We were able to convert the triazene function into a thiophenyl group (30), an azide (31) ${ }^{[19]}$ a bromide (32), an iodide (33), and an arylazo group (34) (Scheme 7).

Finally, we have investigated whether it is possible to use the triazene group in $\mathbf{2 5}$ as a directing group for $\mathrm{C}-\mathrm{H}$ activation reactions. ${ }^{[20]}$

We performed a coupling reaction with benzyl acrylate in the presence of $\left[\mathrm{Cp}^{*} \mathrm{RhCl}_{2}\right]_{2}$ as catalyst precursor and $\mathrm{Cu}(\mathrm{OAc})_{2}$ as oxidant. After work-up, were able to isolate the vinylation product 35 in 58\% yield (Scheme 7). As evidenced by a crystallographic analysis of 35 (see SI), the coupling had occurred adjacent to the pyrazole group and not adjacent to the triazene. Apparently, the directing abilities of the pyrazole are superior to that of the triazene function. Still, this type of reaction allows for further diversification of the indazolo[2,3-a]quinolone moiety.

\section{Conclusion}

The utilization of alkynyl triazenes as substrates in transition metal-catalyzed cyclization and annulation reactions represents a conceptually new way to synthesize aromatic triazenes. To demonstrate the feasibility of this approach, we have investigated $\mathrm{Rh}^{\mathrm{III}}$ catalyzed annulation reactions with alkynyl triazenes. Oxidative coupling reactions were realized for phenylsubstituted indoles, imidazoles and sydnones. The best yields were observed for phenylsydnone. For the other substrates, the yields were compromised to some extend by degradation reaction of the alkynyl triazenes. By comparing the different reactions, it is apparent that mild reactions conditions are key to achieve clean conversions with these acid sensitive alkynes. This sensitivity should be kept in kind for future studies in this area.

An intrinsic advantage of using alkynyl triazenes in cyclization/annulation reactions is the possibility to employ the products for further synthetic transformations. In the case of the annulation products derived from phenylsydnone, we were able to use the reactivity of the triazene function and of the sydnone group for subsequent reactions. Notably, it was possible to prepare as series of new indazolo[2,3a]quinolones by substitution of the triazene group.

\section{Experimental Section}

For experimental details, full characterization, crystallographic data (Crystallographic data have been deposited to the CCDC and correspond to the following codes: 6 (1867241), 10a (1867243), 14a (1867244) and 35 (1867242).) and NMR spectra of new compounds, see Supporting Information.

General procedure the Rh-catalyzed reaction of phenylsydnone with alkynyl triazenes.

A suspension of the sydnone $(0.20 \mathrm{mmol}, 1.0$ equiv. $)$, the corresponding alkynyl triazene ( $0.24 \mathrm{mmol}, 1.2$ equiv.), anhydrous copper(II) acetate (73 $\mathrm{mg}, 0.40 \mathrm{mmol}, 2.0$ equiv.) and $\left[\mathrm{Cp}^{*} \mathrm{RhCl}_{2}\right]_{2}(2.5 \mathrm{mg}, 4.0 \mu \mathrm{mol}, 2 \mathrm{~mol} \%)$ in dry acetonitrile (2 mL, $0.1 \mathrm{M})$ was stirred at room temperature for $16 \mathrm{~h}$. Next the reaction mixture was evaporated under reduced pressure and the crude directly purified using flash column chromatography $\left(30 \mathrm{~cm}, \mathrm{SiO}_{2}\right.$, cyclohexane:ethyl acetate gradient from 20:1 to 10:1).

\section{Acknowledgements}

This work was supported by a Marie Curie EPFL Fellows COFUND fellowship (T.W.).

\section{References}

[1] S. Bräse, Acc. Chem. Res. 2004, 37, 805-816.

[2] a) D. Wang, S. Cui, Tetrahedron 2016, 72, 2725-2730; b) C. Wang, H. Chen, Z. Wang, J. Chen, Y. Huang, Angew. Chem., Int. Ed. 2012, 51, 7242-7245; c) C. Wang, H. Sun, Y. Fang, Y. Huang, Angew. Chem., Int. Ed. 2013, 52, 5795-5798; d) M. Zhang, Y. Zhang, X. Jie, H. Zhao, G. Li, W. Su, Org. Chem. Front. 2014, 1, 843-895; e) L. $\mathrm{Xu}$, W. Yang, L. Zhang, M. Miao, Z. Yang, X. Xu, H. Ren, J. Org. Chem. 2014, 79, 9206-9221; f) H. Sun, Y. Huang, Synlett 2015, 26, 2751-2762.

[3] K. C. Nicolaou, C. N. C. Boddy, S. Bräse, N. Winssinger, Angew. Chem., Int. Ed. 1999, 38, 2096-2152.

[4] a) D. B. Kimball, M. M. Haley, Angew. Chem., Int. Ed. 2002, 41, 3338-3351; b) D. K. Kölmel, N. Jung, S. Bräse, Aust. J. Chem. 2014, 67, 328-336; c) Y. Zhang, D. Cao, W. Liu, H. Hu, X. Zhang, C. Liu, Curr. Org. Chem. 2015, 19, 151-178.

[5] a) F. Marchesi, M. Turriziani, G. Tortorelli, G. Avvisati, F. Torino, L. De Vecchis, Pharmacol. Res. 2007, 56, 275-287; b) Q. Lei, S. Zhang, M. Liu, J. Li, X. Zhang, Y. Long, Mol. Divers. 2017, 21, 957-966.

[6] M. J. Wanner, M. Koch, G.-J. Koomen, J. Med. Chem. 2004, 47, 6875-6883.

[7] a) D. H. Sieh, D. J. Wilbur, C. J. Michejda, J. Am. Chem. Soc. 1980, 102, 3883-3887; b) D. H. Sieh, C. J. Michejda, J. Am. Chem. Soc. 1981, 103, 442-445; c) A. A. Suleymanov, R. Scopelliti, F. Fadaei Tirani, K. Severin, Org. Lett. 2018, 20, 3323-3326.

[8] G. Kiefer, T. Riedel, P. J. Dyson, R. Scopelliti, K. Severin, Angew. Chem., Int. Ed. 2015, 54, 302-305.

[9] a) F. G. Perrin, G. Kiefer, L. Jeanbourquin, S. Racine, D. Perrotta, J. Waser, R. Scopelliti, K. Severin, Angew. Chem., Int. Ed. 2015, 54, 13393-13396; b) D. Kossler, F. 
G. Perrin, A. A. Suleymanov, G. Kiefer, R. Scopelliti, K. Severin, N. Cramer, Angew. Chem., Int. Ed. 2017, 56, 11490-11493; c) L. N. Jeanbourquin, R. Scopelliti, F. Tirani Fadaei, K. Severin, Helv. Chim. Acta 2017, 100, e1700186; d) L. N. Jeanbourquin, R. Scopelliti, F. Fadaei Tirani, K. Severin, Org. Lett. 2017, 19, 2070-2073.

[10] a) S.-S. Li, L. Qin, L. Dong, Org. Biomol. Chem. 2016, 14, 4554-4570; b) G. Song, F. Wang, X. Li, Chem. Soc. Rev. 2012, 41, 3651-3678; c) J. Wencel-Delord, F. W. Patureau, F. Glorius, Top. Organomet. Chem. 2016, 55, 1-27; d) S.-S. Li, L. Qin, L. Dong, Org. Biomol. Chem. 2016, 14, 4554-4570; e) T. Satoh, M. Miura, Chem. - Eur. J. 2010, 16, 11212-11222.

[11] a) H. Ji-Rong, D. Lin, H. Bo, P. Cheng, C. Ying-Chun, Chem. Eur. J. 2012, 18, 8896-8900; b) Q. Ge, B. Li, B. Wang, Org. Biomol. Chem. 2016, 14, 1814-1821.

[12] a) P. C. Too, S. Chiba, e-EROS, John Wiley \& Sons, Ltd., 2013, pp. 1-6; b) P. M. Maitlis, Acc. Chem. Res. 1978, 11, 301-307.

[13] K. Janíková, L. Jedinák, T. Volná, P. Cankar̆, Tetrahedron 2018, 74, 606-617.

[14] a) K. Morimoto, K. Hirano, T. Satoh, M. Miura, Org. Lett. 2010, 12, 2068-2071; b) X. Yang, Y. Li, L. Kong, X. Li, Org. Lett. 2018, 20, 1957-1960.

[15] L. Li, H. Wang, X. Yang, L. Kong, F. Wang, X. Li, J. Org. Chem. 2016, 81, 12038-12045.

[16] a) E. Decuypère, L. Plougastel, D. Audisio, F. Taran, Chem. Commun. 2017, 53, 11515-11527; b) D. L. Browne, J. P. A. Harrity, Tetrahedron 2010, 66, 553-568.

[17] V. Hladíková, J. Váňa, J. Hanusek, Beilstein J. Org. Chem. 2018, 14, 1317-1348.

[18] K. K. Sun, Tetrahedron Lett. 1986, 27, 317-320.

[19] C.-Y. Liu, P. Knochel, J. Org. Chem. 2007, 72, 71067115.

[20] a) G. Song, X. Li, Acc. Chem. Res. 2015, 48, 10071020; b) R.-Y. Zhu, M. E. Farmer, Y.-Q. Chen, J.-Q. Yu, Angew. Chem., Int. Ed. 2016, 55, 10578-10599; c) M. R. Yadav, R. K. Rit, M. Shankar, A. K. Sahoo, Asian J. Org. Chem. 2015, 4, 846-864; d) M. C. Henry, M. A. B. Mostafa, A. Sutherland, Synthesis 2017, 49, 4586-4598; e) Y. Yang, K. Li, Y. Cheng, D. Wan, M. Li, J. You, Chem. Commun. 2016, 52, 2872-2884; f) C. Sambiagio, D. Schönbauer, R. Blieck, T. Dao-Huy, G. Pototschnig, P. Schaaf, T. Wiesinger, M. F. Zia, J. Wencel-Delord, T. Besset, B. U. W. Maes, M. Schnürch, Chem. Soc. Rev. 2018. 


\section{FULL PAPER}

Synthesis of heteroaryl triazenes via Rh(III)catalyzed annulation reactions with alkynyl triazenes.

Adv. Synth. Catal. Year, Volume, Page - Page

Tim Wezeman, Rosario Scopelliti, Farzaneh Fadaei Tirani and Kay Severin*

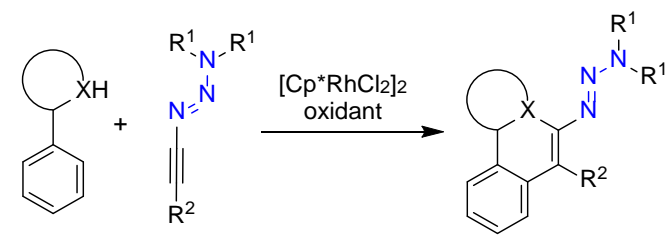

$\mathrm{X}=\mathrm{N}$ or $\mathrm{C}$ 